\title{
Transmissible gastroenteritis virus nsp7 protein localized in the cytoplasm down-regulates interleukin 8 expression in porcine intestinal epithelial cell
}

\author{
Q. ZHANG", J. L. HUANG*, Y. B. LIANG*, Y. P. HE, D. W. TONG*, X. G. XU* \\ College of Veterinary Medicine, Northwest A\&F University, Yangling, Shaanxi 712100, P. R. China
}

Received November 11, 2016; revised May 30, 2017; accepted January 9, 2018

\begin{abstract}
Summary. - Transmissible gastroenteritis virus (TGEV) is an important pathogen in swine that is responsible for substantial economic losses. Previous studies suggest that the TGEV non-structural protein 7 (nsp7) plays an important role in the viral assembly process. However, the subcellular localization and other functions of the TGEV nsp7 protein are still unclear. In this study we have examined the subcellular localization and other functions of TGEV nsp7 protein through analysis of its effects on cell growth, cell cycle progression, interleukin 8 (IL-8) expression, and NF- $\mathrm{KB}$ activation. Our results showed that the nsp7 protein is localized in the cytoplasm and has no effect on intestinal epithelial cells (IECs) growth, cell cycle, and cyclin A expression. Further studies showed that TGEV nsp7 protein had no effect on GRP78 expression, could not induce endoplasmic reticulum (ER) stress and activate NF- $\kappa \mathrm{B}$ activity. Interestingly, the IECs expressing nsp7 protein secreted lower levels of IL-8 than control cells. This is the first report to demonstrate the subcellular localization and novel functions of TGEV nsp7 protein. These findings provide novel information about the function of the poorly characterized TGEV non-structural protein 7.
\end{abstract}

Keywords: transmissible gastroenteritis virus; nsp7 protein; endoplasmatic reticulum stress; interleukin 8; $\mathrm{NF}-\kappa \mathrm{B}$

\section{Introduction}

Transmissible gastroenteritis (TGE) is a fatal and highly contagious enteric infection of swine, which is characterized by vomiting, and severe watery diarrhea, associated with high morbidity in swine of all ages and high mortality in neonatal piglets (Wesley et al., 1991). The causative agent of TGE is transmissible gastroenteritis virus (TGEV). TGEV belongs to the genus Coronavirus, the family Coronaviridae. TGEV like other coronaviruses is a pleomorphic enveloped virus that contains a large, single-stranded, positive-sense $28.5 \mathrm{~kb}$ RNA genome (Wesley et al., 1990). Genome contains a 5' cap and a poly (A) tail at $3^{\prime}$ end and has 7 open reading frames

*Corresponding authors. E-mail: dwtong@nwsuaf.edu.cn, tiger2003@ nwsuaf.edu.cn; phone: +86-29-8709-1117. \#These authors contributed equally to this work.

Abbreviations: $\mathrm{ER}=$ endoplasmic reticulum; IEC $=$ intestinal epithelial cell line; IL-8 = interleukin 8 ; nsp7 = non-structural protein 7 ; ORFs $=$ open reading frames; $\mathrm{TGE}=$ transmissible gastroenteritis; TGEV $=$ TGE virus
(ORFs) (Hiscox et al., 1995). The genes of TGEV are arranged in the order of 5'-Rep-S-3a-3b-E-M-N-7-3'. Four structural proteins include spike protein $(\mathrm{S})$, membrane protein $(\mathrm{M})$, envelope protein $(\mathrm{E})$, and nucleoprotein $(\mathrm{N})$; the other four proteins, Rep, 3a, 3b and 7 are non-structural proteins (Ribes et al., 2011; Lan et al., 2011). About two-thirds of the entire RNA comprises of ORFs $1 \mathrm{a}$ and $1 \mathrm{~b}$, encoding the replicase (Eleouet et al., 1995).

The functions of four structural proteins have been intensively studied (Eleouet et al., 2000; Godet et al., 1992). As for TGEV non-structural proteins, it has been reported that TGEV non-structural protein 7 (nsp7) is encoded by 237 bp long open reading frame 7 (ORF7) at the 3' end of the viral genome. The nsp7 is 78 amino acids long, hydrophobic protein with molecular weight of $9.1 \mathrm{kDa}$ (Tung et al., 1992) expressed during virus replication, which is associated with the endoplasmic reticulum and cell surface membrane of infected cells. TGEV gene 7 is not an essential gene for virus viability but its deletion influences replication and attenuates virus virulence in piglets (Ortego et al., 2003; 
Park et al., 2008). According to Snijders study, coronavirus nsp7 to nsp16, the cleavage products of two large replicase polyproteins translated from the virus genome, can direct coronavirus RNA synthesis and processing with nsp7 to nsp10 acting as crucial cofactors of these enzymes (Snijder et al., 2016). Additionally, some reports show that the deletion of gene 7 in feline coronavirus (FCoVs), another member of the Coronavidae family, attenuated the viral virulence (Herrewegh et al., 1995; Kennedy et al., 2001). The nsp7 in porcine reproductive and respiratory syndrome virus (PRRSV), member of the Nidovirales order, can facilitate humoral immune system to produce anti-nsp7 antibodies, which are highly expressed in PRRSV-infected swine. Despite the studies mentioned above, the functions of TGEV nonstructural proteins still requires further research.

Recently, no data are reported about the subcellular localization of TGEV nsp7 protein, its effects on cell growth and cell cycle progression. The intestine is the target organ of TGEV in piglets (Hooper et al., 1966; Cornelius et al., 1968). The porcine intestinal epithelial cells (IECs) are the target cells of TGEV. The epithelial cells in the gut serve as a physical barrier, restricting the movement of components and the passage of potentially harmful microorganisms between the lumen and the underlying mucosa (Schierack et al., 2006).

In this study, we have analyzed the subcellular localization of TGEV nsp7 and elucidated the effects and mechanisms of this protein on cell cycle and cell growth. The results show that the expression of TGEV nsp7 has no effect on cell growth, cell cycle and cyclin A expression in IEC. Furthermore, we have shown that TGEV nsp7 is localized in the cytoplasm and does not induce ER stress. Our study also showed that the degradation of TGEV nsp7 is not via the proteasome pathway. In addition, the results also show that TGEV nsp7 protein can down-regulate IL-8 expression. These results have potentially important implications for understanding the molecular mechanisms of TGEV pathogenesis. To our knowledge, this is the first report about TGEV nsp7 protein function in porcine intestinal epithelial cells.

\section{Materials and Methods}

Vectors, plasmids, and cells. The pEGFP-N1 eukaryotic expression vector was purchased from Clontech (USA) and Escherichia coli DH5a used for cloning were purchased from Tiangen Biotech (China). In this study, the TGEV Shannxi strain was isolated from intestinal tract contents of TGEV infected piglets in Shannxi Province of China and nsp7 gene of TGEV was amplified as described previously (Ding L et al., 2011). The IECs which were kindly provided by Prof. Yan-Ming Zhang, College of Veterinary Medicine, Northwest A\&F University, were cultured as described previously (Wang et al., 2010). IECs were grown in Dulbecco's modified eagle medium (DMEM) (Gibco BRL, USA) supplemented with 10\% heat-inactivated new born calf serum (FCS, Gibco BRL, USA), $100 \mathrm{IU}$ of penicillin and $100 \mu \mathrm{g} / \mathrm{ml}$ of streptomycin at $37^{\circ} \mathrm{C}$ in a $5 \% \mathrm{CO}_{2}$ atmosphere incubator. The culture medium was replaced every 3 days.

Antibodies and reagents. Mouse monoclonal antibodies against GRP78, cyclin A, NF- $\mathrm{kB}$ p65, and $\beta$-actin were purchased from Santa Cruz Biotechnology (Santa Cruz, Inc., USA), Horseradish peroxidase (HRP)-conjugated secondary antibody was purchased from Pierce (Pierce, USA). The MG132 proteasome inhibitor was purchased from Calbiochem (USA) and the nuclear staining dye Hoechest 33342 and ER-Tracker ${ }^{\mathrm{TM}}$ Red probe were obtained from Invitrogen (USA).

Construction of recombinant plasmid. The primers used for TGEV ORF7amplification were as follows: forwards primer (TGEVXhoI), 5'-CCG CTCGAGATGCTCGTCCTCCTCCATGC-3' (28032-28051 of TGEV TS strain) and reverse primer (TGEVEcoRI), 5'-CGGGAATTCCGTGGTGGTGGTGGTGGTGCAT AATATGACTAACAATC-3' (28249-28268 of TGEV TS strain). The restriction sites are underlined. The reverse primer contained six his tags. The primers were designed according to the archived TGEV TS strain nucleotide sequence (GenBank: DQ201447.1) and synthesized by Shanghai Invitrogen (China). The PCR products were separated on 1.0\% agarose gel electrophoresis, purified from the gel, digested with restriction enzymes and cloned into the pEGFP-N1 expression vector. The recombinant plasmid was designated as p7-EGFP and recovered from transformed E. coli using a Plasmid mini-kit (Axygen, China). Plasmids were verified by enzyme digestion and DNA sequencing.

Transfection. IECs were seed into 6-well plates $24 \mathrm{~h}$ before transfection (up to $70-80 \%$ confluence). Cells were transfected with p7-EGFP and pEGFP-N1 control vector using Lipofectamine 2000 (Invitrogen, USA) and maintained in selection media containing $1200 \mu \mathrm{g} / \mathrm{ml} \mathrm{G} 418$ for two weeks.

Nsp7 protein degradation characteristics. The cell lines expressing p7-GFP protein and GFP were seeded into 6-well plates at a suitable concentration. After incubation at $37^{\circ} \mathrm{C}$ with $5 \% \mathrm{CO}_{2}$ for $24 \mathrm{~h}$, the culture medium was replaced with fresh medium containing $20 \mu \mathrm{mol} / \mathrm{l} \mathrm{MG} 132$, and then incubated in a $\mathrm{CO}_{2}$ incubator at $37^{\circ} \mathrm{C}$ for $24 \mathrm{~h}$. After $24 \mathrm{~h}$ incubation, the cells were washed twice with PBS and incubated with Hoechst 33342 at $37^{\circ} \mathrm{C}$ for $15 \mathrm{~min}$. Images were analyzed by Fluorescence microscope (Model TE2000, Nikon, Japan).

Confocal microscopy analysis. To examine the expression and subcellular localization of TGEV nsp7 protein, the stable cell lines expressing p7-GFP protein or control cells (GFP and untransfected cells) were grown on glass cover slips. Cells washed with PBS were incubated with Hoechst 33342 at $37^{\circ} \mathrm{C}$ for $25 \mathrm{~min}$, washed with PBS and incubated with ER Tracker Red probe (Invitrogen, USA) at $37^{\circ} \mathrm{C}$ for $25 \mathrm{~min}$ and washed with PBS again. Images were viewed by laser confocal scanning microscopy (Model LSM510 META, Zeiss, Germany).

Western blot analysis. Cells were harvested and washed with icecold PBS, then treated with ice-cold RIPA lysis buffer with $1 \mathrm{mmol} / \mathrm{l}$ 
Table 1. The primers for real-time PCR

\begin{tabular}{lllll}
\hline Gene & Forward primer (5'-3') & Reverse primer (5'-3') & Product (bp) & Acc. No. \\
\hline Cyclin A & AAGTTTGATAGATGCTGACCCGTAC & GCTGTGGTGCTCTGAGGTAGGT & 194 & 114 \\
GRP78 & AATGGCCGTGTGGAGATCA & GAGCTGGTTCTTGGCTGCAT & GQ265874 \\
IL-8 & CTGGCTGTTGCCTTCTTG & TCGTGGAATGCGTATTTATG & 113 & 150 \\
Bcl-2 & TTGTGGCCTTCTTTGAGTTCG & CTACCCAGCCTCCGTTATCC & M86923 \\
$\beta$-actin & GGACTTCGAGCAGGAGATGG & AGGAAGGAGGGCTGGAAGAG & 138 & XM_003121700.1 \\
\hline
\end{tabular}

phenylmethyl sulfonfluoride (PMSF). Cell lysates were centrifuged at $12,000 \times \mathrm{g}$ at $4^{\circ} \mathrm{C}$ for $10 \mathrm{~min}$. Protein concentrations were measured by BCA protein assay (Pierce, USA). Equivalent amounts of proteins were loaded and separated on $8-12 \%$ SDS-PAGE. Subsequently, proteins were transferred to PVDF membranes (Millipore Corp, USA). The membranes were blocked with $5 \%$ non-fat milk at room temperature for $1 \mathrm{~h}$, and then incubated with specific primary antibodies over night at $4^{\circ} \mathrm{C}$, followed by incubation with HRP-conjugated secondary antibody at room temperature for $1 \mathrm{~h}$. The signal was detected by enhanced chemiluminescence (ECL) reagents (Pierce, USA).

Cell proliferation assay. The MTT cell proliferation assay was performed to determine the growth properties of TGEV nsp7 expressing and control cells according to the manufacturer's instructions. Briefly, cells were seeded in 96-well culture plates at a concentration of $4 \times 10^{3}$ cells per well in $200 \mu$ of culture medium. After incubation at $37^{\circ} \mathrm{C}$ with $5 \% \mathrm{CO}_{2}$ for $24 \mathrm{~h}, 48 \mathrm{~h}, 72 \mathrm{~h}$ and $96 \mathrm{~h}$, the culture medium was carefully replaced with $200 \mu \mathrm{l}$ of a fresh medium without disturbing the cells. $20 \mu$ of 3-(4, 5-dimethylthiazol-2-yl) 2, 5-diphenylte-trazolium bromide (MTT, $5 \mathrm{mg} / \mathrm{ml}$ ) (Sigma, USA) reagent was added to each well and incubated in a $\mathrm{CO}_{2}$ incubator at $37^{\circ} \mathrm{C}$ for $4 \mathrm{~h}$. After $4 \mathrm{~h}$ incubation, the reactions were stopped by addition of $100 \mu \mathrm{l}$ of DMSO into each well. The absorbance at wavelength of $490 \mathrm{~nm}\left(\mathrm{~A}_{490}\right)$ was read by a microplate reader (Model 680 , Bio-Rad, USA) at appropriate time intervals. The experiments were independently repeated three times.

Cell cycle analysis by flow cytometry. The cell cycle was analyzed by using propidium iodide (PI) staining. Briefly, approximately $2 \times 10^{6}$ cells of the p7-GFP expressing cell line and control cells were treated with trypsin, washed with PBS, resuspended in $75 \%$ ethanol and fixed at $4^{\circ} \mathrm{C}$ for 3 days. Cells were washed with PBS and resuspended in PBS containing $20 \mu \mathrm{g} / \mathrm{ml}$ of RNase A and 50 $\mu \mathrm{g} / \mathrm{ml}$ of PI and incubated at $4^{\circ} \mathrm{C}$ for $30 \mathrm{~min}$ in the dark. Finally, the nuclear DNA content was determined by a Coulter Epics XL flow cytometer (Beckman Coulter, USA).

Real-time quantitative PCR analysis. Total RNA was extracted from cells using Trizol reagent (Invitrogen, USA) according to the manufacturer's instructions and reverse transcribed with M-MLV reverse transcriptase (Thermo Fisher Scientific, USA), oligo(dT) 18 primers and $2 \mu \mathrm{g}$ of total RNA. The expression of genes was quantified using Bio-Rad iQ5 Real Time PCR system (USA). The primers for qPCR used in this study are shown in the Table 1. Reactions were carried out in $25 \mu$ l volume containing SYBR Premix Ex Taq
TM II (Takara, China), sense and anti-sense primers $(0.4 \mu \mathrm{mol} / \mathrm{l})$ and target cDNA (4 ng). The cycling conditions were $95^{\circ} \mathrm{C}$ for 5 min, followed by 40 cycles of $95^{\circ} \mathrm{C}$ for $5 \mathrm{~s}, 60^{\circ} \mathrm{C}$ for $30 \mathrm{~s}$. A negative control was included in each run and specificity of amplification reactions was checked by melting curve (Tm value) analysis. The individual samples were normalized for genome equivalents using the respective CT value for the $\beta$-actin housekeeping gene. The relative quantification of gene expression was analyzed by the two-ddCt method as described previously (Livak et al., 2001).

Detection of NF- $\kappa$ B activity. To determine the alteration of NF- $\kappa \mathrm{B}$ activity by GFP and p7-GFP proteins in IECs, the level of NF- $\kappa B$ activity was measured using the NF- $\kappa$ B p65 TransAM kit (Active Motif) according to the manufacturer's instructions. Briefly, nuclear extracts were prepared by using the Nuclear extract kit (KeyGEN, China) and protein concentrations were measured by the BCA protein assay. Lysates ( $50 \mu \mathrm{g}$ total proteins) were incubated in 96well plates coated with the oligo-nucleotide probes recognized by active p65. Protein 65 was detected by a specific antibody against p65, followed by a horseradish peroxidase (HRP)-conjugated secondary antibody. The colorimetric reaction was measured at 450 $\mathrm{nm}\left(\mathrm{A}_{450}\right)$. This experiment was repeated three times.

Enzyme-linked immunosorbent assay (ELISA). The stable TGEV ORF7 gene expressing cells and the control cells were seeded in 24well plates at a density of $1 \times 10^{5}$ cells $/ \mathrm{ml}$ in DMEM with $10 \%$ FCS and cultured for $48 \mathrm{~h}$. The culture medium was then collected and centrifuged in a microcentrifuge at $1000 \times \mathrm{g}$ for $5 \mathrm{~min}$ to remove debris; the supernatants were then frozen at $-80^{\circ} \mathrm{C}$ until analyzed. The concentrations of IL- 8 were measured using a swine IL-8 ELISA kit according to the manufacturer's instructions (Invitrogen, USA).

Statistical analysis. Data are shown as the means \pm SD of three independent experiments done in triplicate. For each assay, student's $t$-test was used for statistical comparison. A value of $P<0.05$ was considered significant.

\section{Results}

\section{Confirmation of TGEV nsp7 protein expression in IEC}

Western blot analyses showed that the cells transfected with p7-EGFP and pEGFP-N1 plasmid expressed protein of molecular mass of approximately $36 \mathrm{kDa}$ and $27 \mathrm{kDa}$ that were detected by anti-EGFP monoclonal antibodies (Fig. 1). 


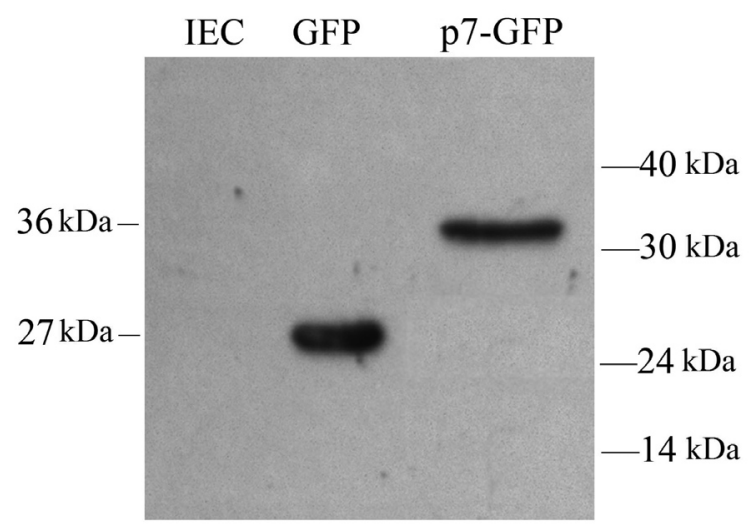

Fig. 1

Detection of p7-GFP fusion protein expression

Western blot analyses of whole extracts of cell lines expressing p7-GFP protein and control IECs. The proteins were detected by anti-GFP antibody.

Since the molecular mass of GFP is known to be approximately $27 \mathrm{kDa}$, this is in an agreement with the predicted size of the nsp7 protein. However, there was no signal in negative IEC control cells.
TGEV nsp7 protein degradation characteristics

The degradation characteristic of nsp7 protein was investigated using fluorescence microscope. The results showed that $\mathrm{p} 7-\mathrm{GFP}$ protein as well as GFP protein expression had no difference in the cell lines treated with MG132 and untreated cells (Fig. 2). The results showed that TGEV nsp7 protein degradation could not be inhibited by MG132 proteasome inhibitor and that the nsp7 protein degradation was not by the proteasome pathway.

\section{TGEV nsp7 protein subcellular localization}

The subcellular localization of nsp7 protein was investigated by confocal fluorescence microscopy. The results showed that $\mathrm{p} 7$-GFP protein was localized in the cytoplasm. By contrast, the GFP protein was distributed throughout the whole cell (Fig. 3).

TGEV nsp7 protein has no influence on cell proliferation

Compared with the GFP protein expressing and the control cells, the nsp7 protein expressing cells, showed a slight change in mitotic activity which caused only low change

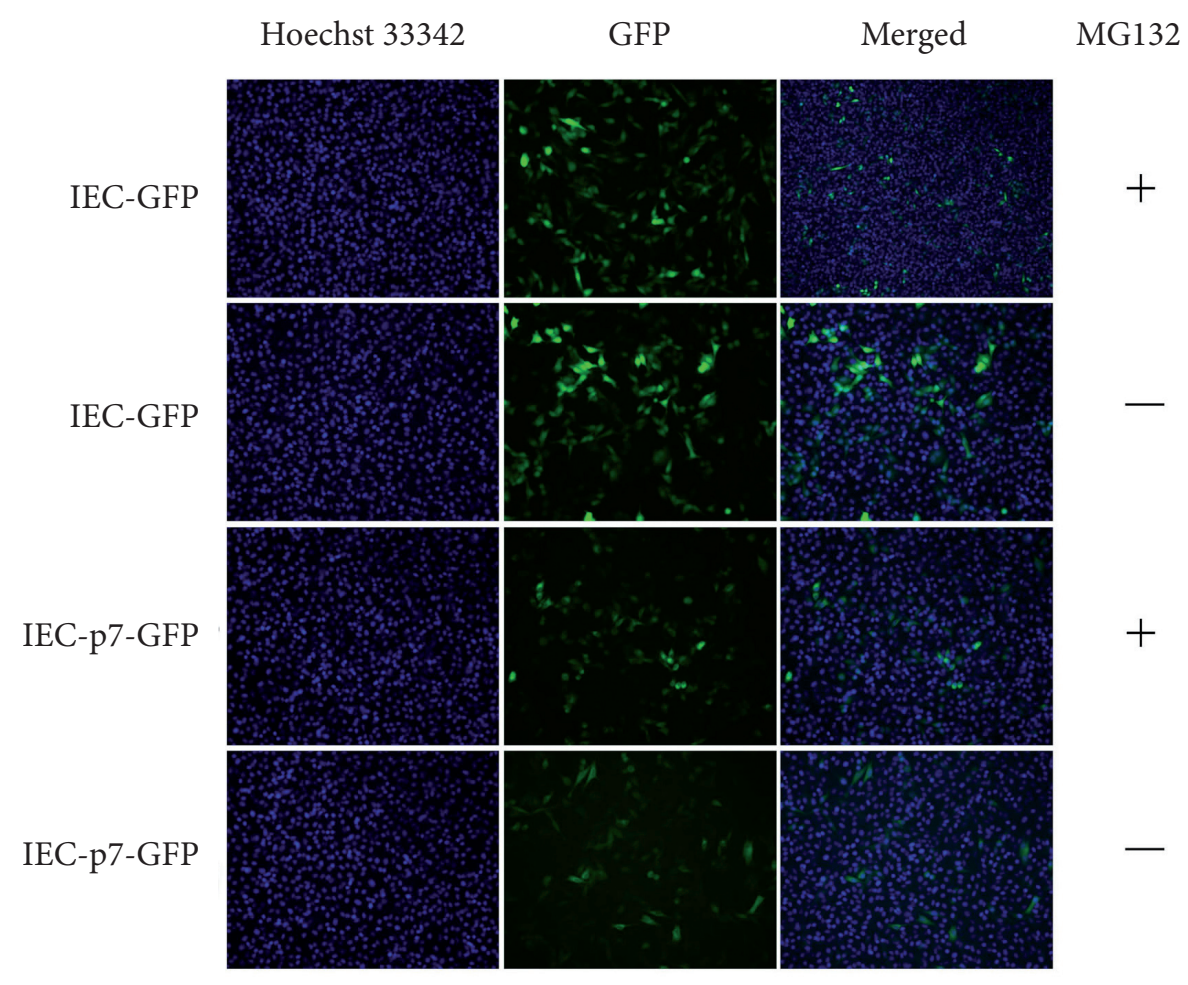

Fig. 2

Fluorescence detection of GFP and p7-GFP fusion protein expressed in IECs $(100 \times)$

All the cell lines were stained by Hoechst 33342 . The images show that expression of p7-GFP in IECs treated with MG132 is the same as in untreated cells. 


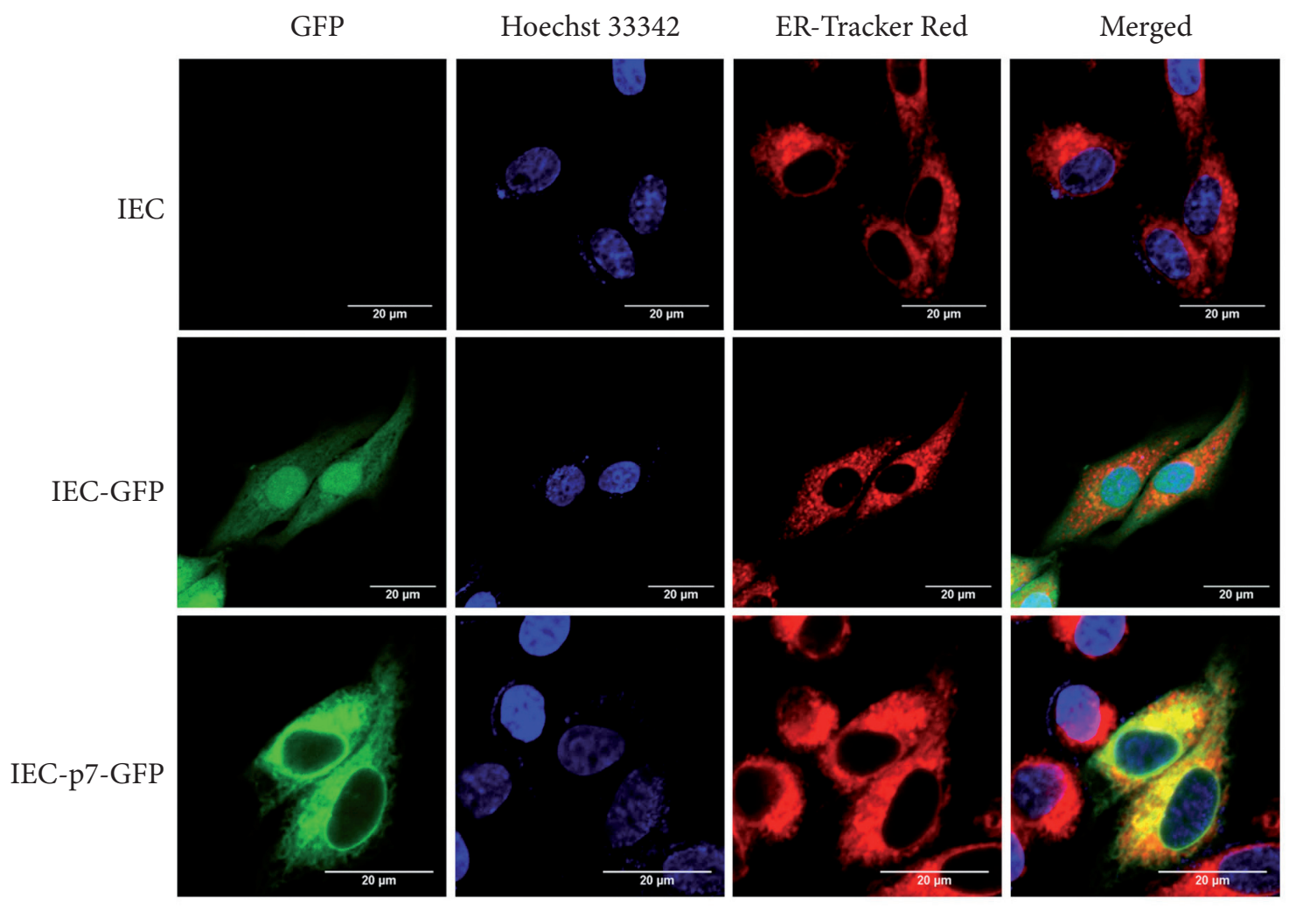

Fig. 3

Subcellular localization of p7-GFP fusion protein in IECs by confocal microscopy

All the cell lines were stained by Hoechst 33342 and ER-Tracker ${ }^{\text {tax }}$ Red. Merged images show co-localization of p7-GFP protein in the ER. Bar $=20 \mu \mathrm{m}$.

in cell number. The results suggest that cell proliferation of nsp7 protein expressing cells did not change over a time course (Fig. 4).

\section{TGEV nsp7 protein does not affect cell cycle}

To investigate whether TGEV nsp7 expression has effect on cell cycle, flow cytometric analysis was performed based on DNA content in nuclei stained with PI. The proportions of G0/G1 phase, S-phase and G2/M phases for the control cells were $54 \%, 37.89 \%$ and $8.11 \%$, respectively. For IECs expressing GFP, the proportions of the phases were G0/G1: 56.22\%, S-phase: $38.01 \%$ and G2/M phase: $5.76 \%$, whereas for $\mathrm{p} 7-\mathrm{GFP}$ expressing IECs, the proportions were G0/G1: $55.18 \%$, S-phase: $35.49 \%$ and G2/M phase: $9.33 \%$. The histograms were quantitatively analyzed according to the data above to determine the percentage of cells in G0/G1, $\mathrm{S}$, and G2/M phases, where G0/G1 phase cells show $2 \mathrm{~N}$ DNA content and G2/M phase cells show 4 N DNA content (Fig. 5). The results showed that relatively to control cells, TGEV nsp7 protein expression caused a slight increase in the number of cells in the each phase. Taken together,

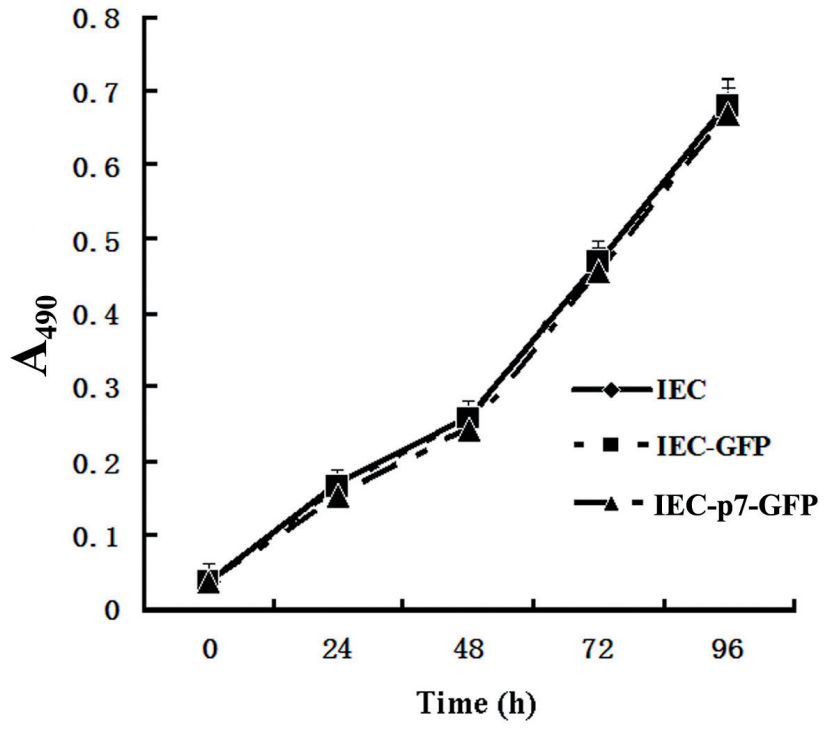

Fig. 4

Cell proliferation assay of the IECs expressing nsp7 The MTT assay was used to measure proliferation of IECs during $96 \mathrm{~h}$. Each data set represents the mean \pm SD of six replicates. 


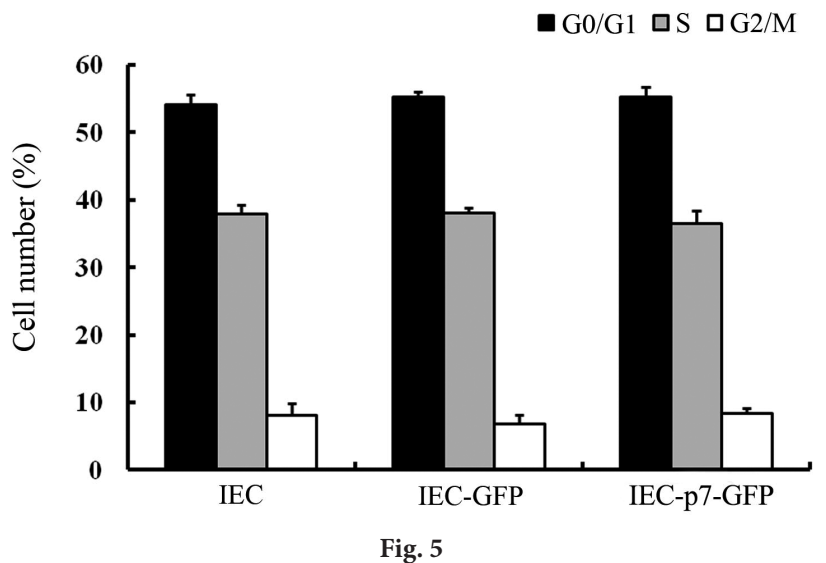

The analysis of cell division stages of IECs expressing TGEV nsp7 protein by flow cytometry

The percentage analysis of the cells in each cell cycle phase. The results are mean \pm SD from three independent experiments.

these results strongly suggested that TGEV nsp7 had no effect on cell cycle.

\section{TGEV nsp7 protein has no effect on cyclin A expression}

One of the key regulators of cell cycle progression from the $\mathrm{S}$ phase to the $\mathrm{G} 2 / \mathrm{M}$ phase is cyclin $\mathrm{A}$. To understand the effect of nsp7 protein expression on cell cycle, we examined cyclin A mRNA expression in transfected and control cells by qPCR. The results showed that there was no significant difference of cyclin A mRNA level between the p7-GFP

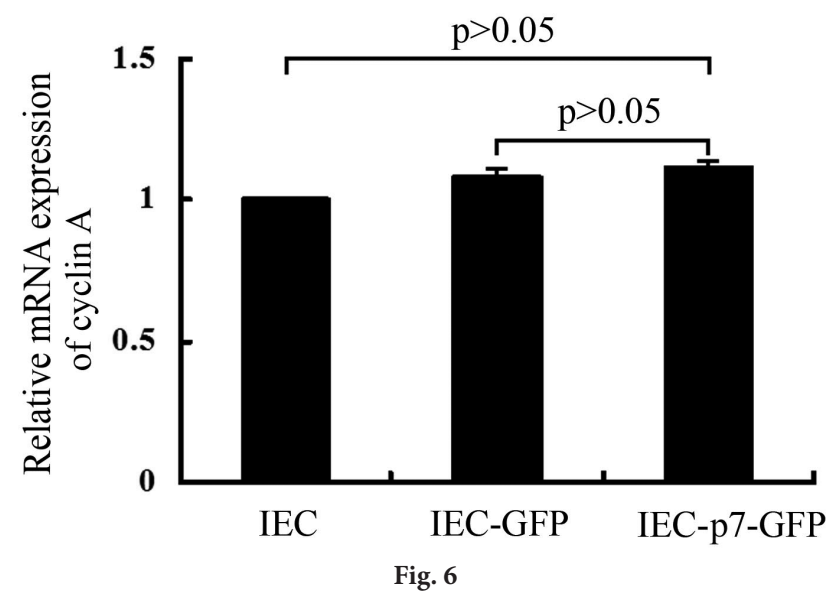

The effect of TGEV nsp7 expression on the cyclin A protein expression Total RNA was extracted from cells expressing GFP alone, p7-GFP or untransfected cells. Cyclin A mRNA levels were normalized to the corresponding CT value of $\beta$-actin mRNA. The results are mean \pm SD from three independent experiments. expressing cells and cells in both control groups (Fig. 6). The western blot assay showed that cyclin A expression level was only slightly increased in cells that expressed nsp7 protein compared with control cells (data not shown), and corresponded with qPCR results, indicating that the expression of nsp7 protein had no significant effect on cyclin A expression $(\mathrm{P}>0.05)$.

\section{TGEV nsp7 has no effect on GRP78 expression and $N F-\kappa B$ activation}

To analyze the expression of glucose regulated protein 78 (GRP78), we chose a well characterized ER chaperone protein that is a marker of ER stress (Hong, 2005; Li et al., 2008; Quinones, de Ridder, and Pizzo, 2008. The GRP78 transcription level detected by qPCR assay showed a slight increase in nsp7 expressing cell line (Fig. 7). The western blot assay also did not show any GRP78 expression level change between the cells that expressed nsp7 protein and cells in both control groups (data not shown), corresponding with the result of qPCR assay. Analysis of the activity of NF- $\kappa \mathrm{B}$ in p7-GFP expressing cell lines demonstrates that NF- $\kappa \mathrm{B}$ was slightly activated compared with control cells (Fig. 8). Together, these analyses show that expression of TGEV nsp7 has no significant influence on GRP78 and NF- $\mathrm{BB}$, suggesting that the TGEV nsp7 has no significant effect on ER stress activation, though the subcellular localization of nsp7 in the cytoplasm.

\section{TGEV nsp7 down-regulates $I L-8$ expression}

Secretion of IL-8 in the supernatant from untransfected and transfected cells was examined using ELISA assay. P7-

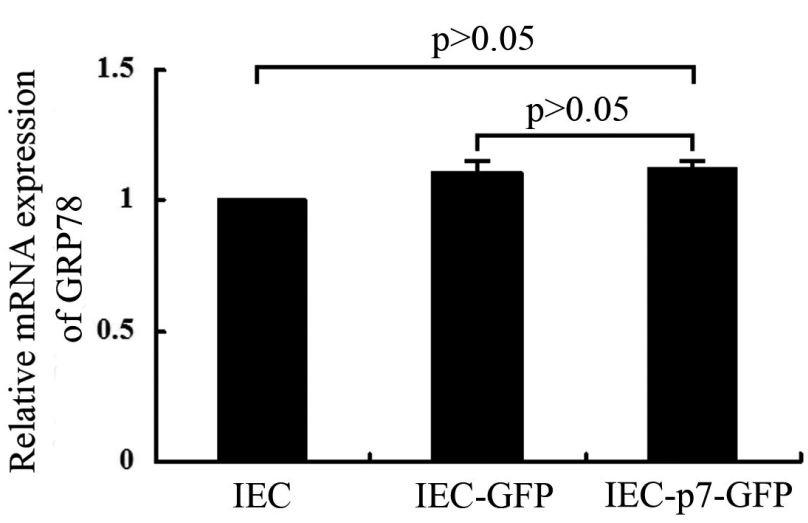

Fig. 7

The effect of TGEV nsp7 expression on the GRP78 expression Total RNA was extracted from cells expressing GFP alone, p7-GFP or untransfected cells. GRP78 mRNA levels were normalized to the corresponding CT value of $\beta$-actin mRNA. The results are mean \pm SD from three independent experiments. 


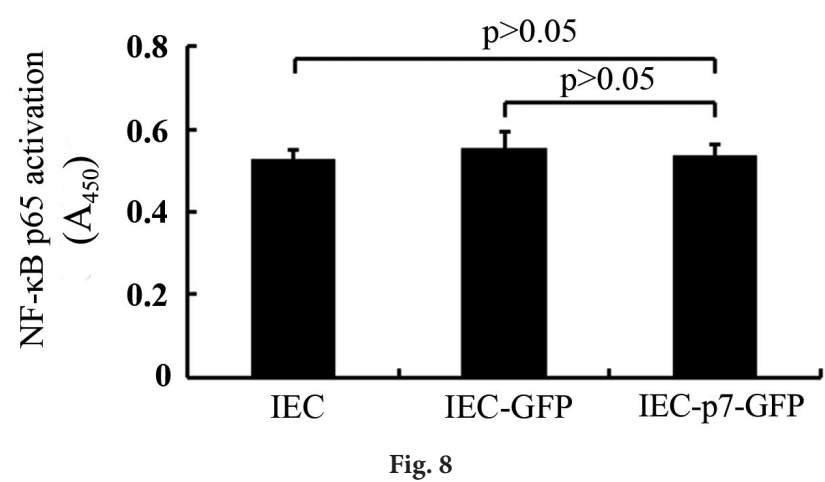

The effect of TGEV nsp7 expression on the NF- $\mathrm{kB}$ activity in IECs NF- $\kappa B$ p 65 activation was determined using the TransAM assay. The data represent the mean and standard deviation from three different experiments.

GFP expressing cells were found to express lower levels of IL-8 compared to GFP expressing cells and the untreated cells (Fig. 9a). Moreover, the mRNA level of IL-8 was detected by qPCR assay and the results showed that TGEV nsp7 expression down-regulated the transcriptional level of IL-8 in comparison with the control cells (Fig. 9b). Thus, these results suggest that TGEV nsp7 expression is responsible for the down-regulation of IL-8 expression.

\section{Discussion}

In recent years, many studies were focused on the function of structural proteins, gene sequence analysis of TGEV, and genetic and phylogenetic analysis based on ORF7 genes. Some of them have reported that ORF7 influences the virulence of TGEV (Ortego et al., 2003). Despite this vast amount of research, the subcellular localization and function of the TGEV nsp7 protein still remains unclear. Although some functions of this protein have been studied, the influence on cell cycle by nsp7 protein is still unknown. Moreover, the function of nsp7 protein is yet to be determined, particularly with regard to its effect on host cell physiological changes. In this study, we constructed a eukaryotic expression vector and generated TGEV nsp7-GFP fusion protein expressing cell line that allowed analysis of its properties. Co-localization studies clearly showed that TGEV 7 protein is localized in the cytoplasm. In this study, the effect of TGEV nsp7 protein on IECs proliferation was determined. MTT assay showed that TGEV nsp7 protein had no influence on cell proliferation and cell cycle. Cyclin A is very important for cells in transition from $S$ phase to G2/M phase. QPCR results showed that there was no significant difference of cyclin A mRNA level between the p7-GFP expressing cells and cells in both control groups, which corresponded to the result of western blot. These results suggested that the TGEV nsp7 protein had no significant effect on host cell proliferation and cell cycle.
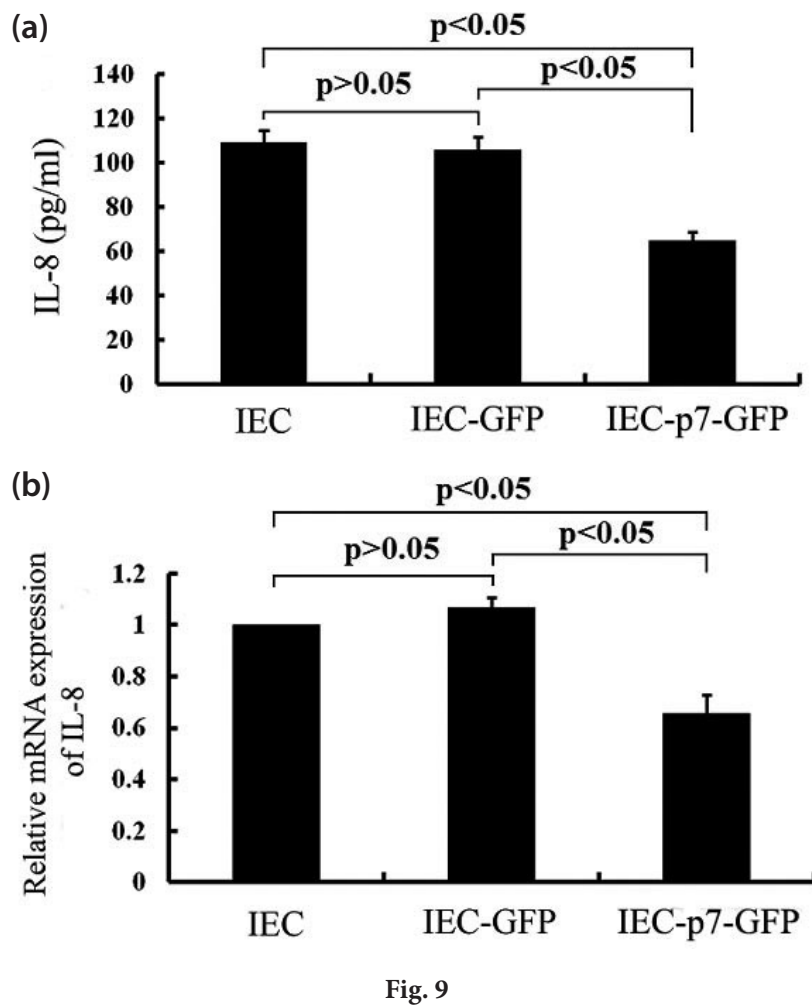

The effect of TGEV nsp7 expression on IL-8 expression (a) The concentrations of IL-8 in p7-GFP expressing cells or control cells supernatants were analyzed by ELISA. Data are mean \pm SD. (b) Effect of TGEV nsp7 expression on porcine IL-8 transcription in IECs. Total RNA was extracted from cells expressing GFP alone, p7-GFP or untransfected cells. IL-8 mRNA levels were normalized to the corresponding CT value of $\beta$-actin mRNA. The results are mean \pm SD.

In this study, it was shown that TGEV nsp7 protein was localized in the cytoplasm and was not able to induce ER stress, as indicated by the low up-regulation of the molecule chaperon GRP78, one of the typical markers of ER stress. The ER plays an essential role in multiple cellular processes that are important for cell survival and normal cellular functions (Anelli et al., 2008). Viruses use the ER as an integral part of their replication strategy, they must contend with the ER stress response and the downstream consequence of ER stress signaling, including the initiation of an inflammatory response through the activation of NF-kB (Park et al., 2008; Herrewegh et al., 1995; Cruz et al., 2011). As a pro-inflammatory neutrophil chemotactic factor, IL-8 plays an important role in the promotion of cell survival signaling and antagonizes the anti-viral activities of interferon. Our previous study suggested that TGEV N protein could induce ER stress and significantly activate NF- $\kappa \mathrm{B}$, which leads to the promotion of IL-8 transcription, while TGEV nsp7 protein as shown in this study could not. In fact, this study showed that TGEV nsp7 protein could even down-regulate the IL-8 expression in IECs. 
There are a number of studies which have revealed that nidovirus nsp7 is involved in the immune interactions between nidoviruses and host cells. For instance, porcine epidemic diarrhea virus (PEDV) nsp7 can inhibit the IFN- $\beta$ and IRF3 promoter activities, thus impairing host cells' innate immune response towards the virus (Zhang Q et al., 2016). However, PRRSV nsp7 was reported to induce autophagy of the host cells, which enhanced cells' innate defense against infection (Cottam et al., 2014). As for TGEV nsp7, it was reported that the absence of TGEV nsp7 led to increased pro-inflammatory responses and acute tissue damage after infection both in vitro and in vivo, with some pro-inflammatory up-regulated genes, such as IL-8 and NF- $\kappa$ B inhibitor alpha (NF- $\mathrm{BIA}$ ) (Cruz et al., 2013). These results showed that the presence of nsp7 reduced inflammatory changes after TGEV infection in both in vitro and in vivo, indicating that TGEV nsp7 could counter-act the host antiviral response and modulate host innate immune response, which are similar to PEDV nsp7 (Cruz et al., 2013). Corresponding to the results above, ours showed that the TGEV nsp7 expression in IECs could not activate NF- $\kappa \mathrm{B}$ activity but could inhibit IL-8 expression on both mRNA and protein level. TGEV nsp7 may inhibit the expression of IL-8 by mechanisms that can be an important part of TGEV immune escape mechanism and requires further research.

One previous study reported that there was no difference of PKR-like endoplasmic reticulum kinase (PERK) activity between cells infected by the TGEV ORF7 deletion mutant virus and the parental virus, which suggests that the deletion of TGEV ORF7 could not induce the ER stress (Cruz et al., 2011). Our observations also showed that nsp7 protein could not influence the GRP78 activity on neither transcription level nor protein level. In conclusion, all data show that TGEV nsp7 has no influence on ER stress response.

To conclude, localized in the cytoplasm but with no influence on GRP78 expression and ER stress, TGEV nsp7 has no effect on the IEC growth, cell cycle and cyclin A expression, either. In addition, though TGEV nsp7 does not influence the NF- $\kappa B$ activity, it can down-regulate the expression level of IL-8. This study provides some new features about the function of the TGEV nsp7 protein which are likely to be very useful in understanding the molecular mechanisms of TGEV pathogenesis.

Acknowledgments. This work was supported by grants from the innovation project for agro-technology of Shaanxi Province (No. 2016NY-095), China. Thank for Professor D. W. Tong providing laboratory conditions.

\section{References}

Anelli T, Sitia R (2008): Protein quality control in the early secretory pathway. EMBO J. 27, 315-327. https://doi.org/10.1038/ sj.emboj. 7601974
Cornelius L, Hooper B, Haelterman E (1968): Changes in fluid and electrolyte balance in baby pigs with transmissible gastroenteritis. Am. J. Vet. Clin. Pathol. 2, 105-113.

Cottam EM, Whelband MC, Wileman T (2014): Coronavirus NSP6 restricts autophagosome expansion. Autophagy 10, 1426-1441. https://doi.org/10.4161/auto.29309

Cruz JL, Sola I, Becares M, Alberca B, Plana J, Enjuanes L, Zuñiga $S$ (2011): Coronavirus gene 7 counteracts host defenses and modulates virus virulence. PLoS. Pathog. 7, 394-397. https://doi.org/10.1371/journal.ppat.1002090

Cruz JL, Becares M, Sola I, Oliveros JC, Enjuanes L, Zúñiga S (2013): Alphacoronavirus protein 7 modulates host innate immune response. J. Virol. 87, 9754-9767. https:// doi.org/10.1128/JVI.01032-13

Eleouet JF, Rasschaert D, Lambert P, Levy L, Vende P, Laude H (1995): Complete sequence (20 kilobases) of the polyprotein-encoding gene 1 of transmissible gastroenteritis virus. Virology 206, 817-822. https://doi.org/10.1006/ viro.1995.1004

Eleouet JF, Slee EA, Saurini F, Castagné N, Poncet D, Garrido C, Solary E, Martin SJ (2000): The viral nucleocapsid protein of transmissible gastroenteritis coronavirus (TGEV) is cleaved by caspase- 6 and 7 during TGEV-induced apoptosis. J. Virol. 74, 3975-3983. https://doi.org/10.1128/ JVI.74.9.3975-3983.2000

Godet M, Haridon RL, Vautherot JF, Laude H (1992): TGEV coronavirus ORF4 encodes a membrane protein that is incorporated into virions. Virology 188, 666-675. https:// doi.org/10.1016/0042-6822(92)90521-P

Herrewegh AAPM, Vennema H, Horzinek MC, Rottier PJM, de Groot RJ (1995): The molecular genetics of feline coronaviruses: comparative sequence analysis of the ORF7a/7b transcription unit of different biotypes. Virology 212, 622-631. https://doi.org/10.1006/viro.1995.1520

Hiscox JA, Cavanagh D, Britton P (1995): Quantification of individual subgenomic mRNA species during replication of the coronavirus transmissible gastroenteritis virus. Virus Res. 36, 119-130. https://doi.org/10.1016/01681702(94)00108-O

Hong M, Lin MY, Huang JM, Baumeister P, Hakre S, Roy A L (2005): Transcriptional Regulation of the Grp78 promoter by endoplasmic reticulum stress: role of TFII-I and its tyrosinephosphorylation. J. Biol. Chem. 280, 16821-16828. https://doi.org/10.1074/jbc.M413753200

Hooper B, Haelterman E (1966): Concepts of pathogenesis and passive immunity in transmissible gastroenteritis of swine. J. Am. Vet. Med. Assoc. 149, 1580-1586.

Kennedy M, Boedeker N, Gibbs P, Kania S (2001): Deletions in the $7 \mathrm{a}$ ORF of feline coronavirus associated with an epidemic of feline infectious peritonitis. Vet. Microbiol. 81, 227234. https://doi.org/10.1016/S0378-1135(01)00354-6

Lan D, Gu J, Xing R, Yuan C, Cui L, Hua X, Yang Z (2011): Sequence analysis of the ORF7 region of chinese transmissible gastroenteritis virus isolate TGEs-1. Bull. Vet. Inst. Pulawy 55, 169-175.

Ding L, Chen GD, Xu XG, Tong DW (2011): Isolation and identification of porcine transmissible gastroenteritis virus Shaanxi 
strain and sequence analysis of its $\mathrm{N}$ gene. Chinese J. Vet. Med. 47, 9-12.

Li J, Ni M, Lee B, Barron E, Hinton DR, Lee AS (2008): The unfolded protein response regulator GRP78/BiP is required for endoplasmic reticulum integrity and stress-induced autophagy in mammalian cells. Cell. Death. Differ. 15, 1460-1471. https://doi.org/10.1038/cdd.2008.81

Livak KJ, Schmittgen TD (2001): Analysis of relative gene expression data using real-time quantitative PCR and the 2(Delta Delta C (T)) method. Methods 25, 402-408. https:// doi.org/10.1006/meth.2001.1262

Ortego J, Sola I, Almazán F, Ceriani JE, Riquelme C, Balasch M, Plana J, Enjuanes L (2003): Transmissible gastroenteritis coronavirus gene 7 is not essential but influences in vivo virus replication and virulence. Virology 308, 13-22. https://doi.org/10.1016/S0042-6822(02)00096-X

Park JH, Han JH, Kwon HM (2008): Sequence analysis of the ORF 7 region of transmissible gastroenteritis viruses isolated in Korea. Virus Genes 36, 71-78. https://doi.org/10.1007/ $\underline{\text { s11262-007-0191-Z }}$

Quinones Q J, de Ridder G G, Pizzo S V (2008): GRP78: a chaperone with diverse roles beyond the endoplasmic reticulum. Histol. Histopathol. 23, 1409-1416.

Ribes JM, Ortego J, Ceriani J, Montava R, Enjuanes L, Buesa J (2011): Transmissible gastroenteritis virus (TGEV)-based vectors with engineered murine tropism express the rotavirus VP7 protein and immunize mice against rotavirus. Virology 410, 107-118. https://doi.org/10.1016/j. virol.2010.10.036
Schierack P, Nordhoff M, Pollmann M, Weyrauch KD, Amasheh S, Lodemann U, Jores J, Tachu B, Kleta S, Blikslager A (2006): Characterization of a porcine intestinal epithelial cell line for in vitro studies of microbial pathogenesis in swine Histochem. Cell Biol. 125, 293-305. https://doi. org/10.1007/s00418-005-0067-Z

Snijder EJ, Decroly E, Ziebuhr J (2016): The Nonstructural Proteins Directing Coronavirus RNA Synthesis and Processing. Adv Virus Res. 96, 59-126. https://doi.org/10.1016/ bs.aivir.2016.08.008

Tung FY, Abraham S, Sethna M, Hung SL, Sethna P, Hogue BG, Brian DA (1992): The 9-kDa hydrophobic protein encoded at the 3 ' end of the porcine transmissible gastroenteritis coronavirus genome is membrane-associated. Virology 186, 676683. https://doi.org/10.1016/0042-6822(92)90034-M

Wang J, Zhang YM, Tong G, Liu F, Zhou H, He L, Yang X, Xu Y, Hong $H$ (2010): The isolation and identification of neonatal swine intestinal epithelial cells. Chinese J. Anim. Vet. Sci. 41, 92-98.

Wesley R, Woods R, Cheung A (1991): Genetic analysis of porcine respiratory coronavirus, an attenuated variant of transmissible gastroenteritis virus. J. Virol. 65, 3369-3373.

Wesley R, Woods R, Cheung A (1990): Genetic basis for the pathogenesis of transmissible gastroenteritis virus. J. Virol. 64, 4761-4766.

Zhang Q, Shi K, Yoo D (2016): Suppression of type I interferon production by porcine epidemic diarrhea virus and degradation of CREB-binding protein by nsp 1 . Virology 489 , 252-268. https://doi.org/10.1016/j.virol.2015.12.010 